\title{
COMPARATIVE ANALYSIS OF THE EFFECTS OF TOPICAL ANAESTHETIC AGENTS ON TEAR QUANTITY AND TEAR QUALITY
}

\author{
BY \\ *GEORGE, O. G. AND OMOKHUA, P. O. \\ DEPARTMENT OF OPTOMETRY, FACULTY OF LIFE SCIENCES \\ UNIVERSITY OF BENIN, EDO STATE, NIGERIA \\ Email:darasabe@yahoo.com \\ *Corresponding author
}

\begin{abstract}
This study compared the effects of different topical anaesthetic agents on tear quantity and tear quality. To establish this, 30 subjects aged between 18-35years with mean age $23 \pm 2.60$ years and free from ocular and systemic diseases were used. The study population consisted of 15 males and 15 females. The quantity and quality of tears were measured for each subject before and after instillation of topical anaesthetic agents. The anaesthetic agents used were $2 \%$ xylocaine hydrochloride, $0.5 \%$ proparacaine hydrochloride and $0.5 \%$ tetracaine hydrochloride. Schirmer's tear test was used to assess the quantity of tears while tear quality was assessed with Non-Invasive Tear Break-Up-Time (NITBUT). The result obtained showed that $0.5 \%$ proparacaine hydrochloride had a statistically significant decrease in tear quantity produced after instillation, using the one way analysis of variance, ANOVA $(\mathrm{F}=4.43, \mathrm{P}<0.005) .0 .5 \%$ tetracaine hydrochloride had a statistically significant effect on tear film stability time $(\mathrm{F}=16.78, \mathrm{P}<0.005)$ while $2 \%$ xylocaine hydrochloride had the least effect on tear quantity and tear quality. Thus, of these three topical anaesthetic agents, $0.5 \%$ tetracaine hydrochloride may be the preferred choice in optometric practice particularly in patients with Keratoconjunctivitis Sicca (KCS) in order to facilitate their tear film stability; while the use of proparacaine should be discouraged in patients with dry eye syndrome.
\end{abstract}

KEYWORDS: $2 \%$ Xylocaine hydrochloride, $0.5 \%$ Proparacaine hydrochloride, $0.5 \%$ Tetracaine hydrochloride, Tear quality, Tear quantity.

\section{Received 25/03/2010}

Accepted 05/06/2010

\section{INTRODUCTION}

"Anaesthetic" comes from the Greek word "anaesthesia" meaning the absence or loss of sensation ${ }^{1}$. Anesthetic acts by temporarily blocking the sensation of pain during diagnostic and therapeutic procedures; this is by inhibiting the influx of sodium ions into the nerve cytoplasm. It binds to the specific receptor site within the sodium channels and blocks the sodium ion movements through this pore. This property blocks the pain sensation locally hence the name local anaesthetic.

Local anaesthetics are made mostly from amino-esters and amino-amides. Proparacaine and tetracaine are the most common forms of aminoesters. Ester anaesthetics produce an almost instantaneous and significant anaesthetic effect when applied to the pain sensitive cornea but they are relatively "short-lived". This is because plasma cholinesterase agents in the blood rapidly metabolize esters. Lidocaine is prototype of amide-linked agents, which are metabolized by liver and are longer acting ${ }^{2}$. Anaesthetics can be given topically, intravenously, intramuscularly, or intrathecally.
Tears are a mixture of secretions from the major and minor accessory lacrimal glands, the goblet cells and the meibomian glands. It covers the anterior surface of the globe and it is about 34$45 \mu \mathrm{m}$ thick. It is composed of 3 layers namely; a superficial lipid layer derived from the secretions of the meibomian glands. This is to retard evaporation of the aqueous layer, a middle aqueous layer secreted by the major and minor lacrimal glands and contains water soluble substances (salts and proteins), a deep mucous layer composed of glycoprotein mucin which overlies the corneal and conjunctival epithelial cells ${ }^{4}$.

The tear layer keeps the surface of the globe moist and serves as a lubricant between the globe and the eyelids. It traps debris and helps remove sloughed epithelial cell. It provides smooth refractive surface for optimum optical function. It contains antibacterial substances lysozyme, betalysin, and $\operatorname{Ig} \mathrm{A}$; to help protect against infection. It helps to maintain corneal hydration by changes in tonicity that occur with evaporation; lastly it is a primary source of atmospheric oxygen for the cornea $^{5,6}$. 
Periodic resurfacing of the tear film is important to prevent dry spots and is accomplished by blinking which is a rapid closure of the eyelid. Blinking occurs involuntarily (reflex blink), as a response to various external stimuli ${ }^{7}$ or voluntarily which involves the movement of upper and lower lids to close the palpebral fissure. The average rate of involuntary blinking is about 12 times/min or once every 4-5 seconds. The inter-blink interval in normal adult humans is 5-10 seconds. The break up time is much longer averaging 30 seconds. The total blink period takes about $0.2-0.3$ seconds but the actual time that the lid is covering the pupil and blocking light from the retina is about 0.13 seconds .

Deficiency in aqueous and mucus component of tears results in rapid break-up time because it gives a constant feeling of ocular irritation or foreign body sensation, and this can result in epithelial damage and occasional corneal ulceration $^{7}$

\section{RESEARCH METHODOLOGY}

Thirty subjects were examined in four weeks clinic visits. In the first week clinic visit, baseline values of their tear quantity and tear quality were measured, using Schirmer's test and NITBUT methods without any anaesthesia. These values served as the control for the 30 subjects. In the second week clinic visit, $2 \%$ xylocaine was instilled in both eyes of the 30 subjects. In the third week clinic visit, $0.5 \%$ proparacaine was instilled in both eyes of the 30 subjects. In the fourth week clinic visit $0.5 \%$ tetracaine was instilled in both eyes of the 30 subjects. Tear quantity and tear quality were measured. In each weekly visit, tear quantity was measured in the right eye while tear quality was assessed using the left eye.

The Schirmer's test was performed by instilling each of the topical anaesthetic into the right eye, and then eye is gently dried of excess tears with a sterile cotton wool. Also tears were removed from the inferior fornix with a filter paper. The room illumination was dimmed. A Schirmer strip folded $5 \mathrm{~mm}$ from one end was inserted at the junction of the middle and outer third of the lower lid taking care not to touch the cornea or lashes. Subjects were asked to keep eyes gently closed and the stop watch was switched on. After 5 minutes, the filter paper was removed and the amount of wetting from the fold was measured and recorded. Less than $10 \mathrm{~mm}$ of wetting after 5 minutes without the use of anaesthesia was considered abnormal while less than $6 \mathrm{~mm}$ with anaesthesia was considered abnormal ${ }^{8}$.

NITBUT was carried out with the use of the Bausch and Lomb Keratometer which was used to measure the Tear Thinning Time or stability of the tear film. The subjects were instructed to put chin on the chin rest and head on the fore head rest with left eye positioned. The keratometer mires were centered and properly aligned. The subject was instructed to blink normally and then stop. The time elapsed from the last complete blink until the mires diffuse or distort was measured using a stopwatch ${ }^{9}$.

\section{RESULTS}

The results of tear volume using these three anaesthetics were analyzed using the mean, the standard deviation and Analysis of Variance (ANOVA). Likewise the results of tear stability using these three anaesthetics were recorded and analyzed using the mean, the standard deviation and the Analysis of Variance (ANOVA). Table 1 shows the results of the mean of tear quantity and tear quality measured using the Schirmer's test and NITBUT test before and after the instillation of the three anaesthetic agents. The mean tear quantity before and after instilling the three types of topical anaesthetic agents $(2 \%$ xylocaine hydrochloride, $0.5 \%$ tetracaine hydrochloride, $0.5 \%$ proparacaine hydrochloride) were shown in table 1 . There was a statistically significant decrease in tear quantity (ANOVA: $\mathrm{F}=4.43, \mathrm{P}<0.005$ ). The source of significance was in mean tear quantity with $0.5 \%$ proparacaine hydrochloride which was $14.07 \pm 7.61 \mathrm{~mm}$, a reduction in mean tear quantity of $7.80 \mathrm{~mm}$ compared to the control which was $21.87 \pm 11.70 \mathrm{~mm}$. The topical anaesthetic that had the least effect in reducing the tear quantity was $2 \%$ xylocaine hydrochloride whose mean tear quantity was $20.13 \pm 9.37 \mathrm{~mm}$, reduction of mean tear quantity of $1.74 \mathrm{~mm}$ compared to the control which was $(21.87 \pm 11.70 \mathrm{~mm})$. The difference between the mean control value and the mean tear quantity of 
$0.5 \%$ tetracaine hydrochloride was $5.77 \mathrm{~mm}$. This was not statistically significant.

The result of the test on tear film stability using the Non-invasive Tear Break up time (NITBUT), showed that the difference in mean NITBUT before and after instilling three different topical anaesthetics was statistically significant (ANOVA: $\mathrm{F}=16.78, \mathrm{P}<0.005)$. The mean NITBUT assessed after the instilling of $0.5 \%$ tetracaine hydrochloride was $33.63 \pm 12.6$ seconds, a mean difference of 11.0 seconds compared with the control value of $22.63 \pm 10.92$ seconds, this was statistically significant $(\mathrm{P}>0.05)$. Whereas the mean difference between control NITBUT and $2 \%$ xylocaine hydrochloride was just 1.23 seconds while it was 6.40 seconds with $0.5 \%$ proparacaine hydrochloride. These were not statistically significant. From these results, the topical anaesthetic that had the least effect on NIBUT was $2 \%$ xylocaine hydrochloride, but $0.5 \%$ tetracaine hydrochloride tended to give a longer duration of tear film stability.

\section{DISCUSSION}

Human tears (secretion and stability) have been a subject of intense study for many years. Many practising optometrists use topical anaesthetic agents to carry out most of their diagnostic examinations. Studies on the effect of these commonly used topical anaesthetics on tear film secretion and stability are limited.

The aim of this study was to compare the effect of different anaesthetic agents on tear quantity and tear stability. The results for tear quantity revealed that $0.5 \%$ proparacaine hydrochloride significantly decreased tear secretion. This confirms that this anaesthetic can affect tear quantity significantly. This study is in agreement with previous study by Lambert et $\mathrm{al}^{9}$, who observed a decrease in mean test values of tear quantity after the instillation of $0.5 \%$ proparacaine hydrochloride. Basal tear secretion increases in response to a variety of irritants applied to the ocular surface or the nasal and oral mucosae. This reflex tear secretion can be prevented by damage to the sensory root of the trigeminal nerve or by topical application of cocaine and other local anaesthetic drugs onto the eye $^{10}$. This suggests that the afferent branch of the tearing response to ocular surface irritation is formed by sensory axons of trigeminal ganglion neurons supplying the eye. The efferent pathway of this reflex is formed by parasympathetic fibres of the superior cervical nerve that act on the main accessory lacrimal glands and meibomian glands to regulate the production of proteins, electrolytes, water and mucin of the tear film ${ }^{10}$. From this, it can be deduced that $0.5 \%$ proparacaine Hydrochloride inhibits parasympathetic stimulation to the main accessory lacrimal glands and meibomian glands more than $0.5 \%$ tetracaine hydrochloride and $2 \%$ xylocaine.

The results for tear stability revealed that $0.5 \%$ tetracaine hydrochloride had a significant increase in tear stability time as compared with the control, $2 \%$ xylocaine and $0.5 \%$ proparacaine hydrochloride. This study is in agreement with Patel et al., who observed an increase in precorneal tear thinning time with $0.5 \%$ tetracaine hydrochloride in brown eyed subjects. It has previously been reported by Cho and Brown ${ }^{10}$ that topical anaesthetic can be used to facilitate tear stability assessment when a subject is unable to refrain from blinking for a sufficient duration of time The result of this study is in agreement with other types of anaesthetics like $0.4 \%$ benoxinate hydrochloride that was used by Cho and Brown ${ }^{10}$, and they observed a significant increase in NITBUT after the instillation of $0.4 \%$ benoxinate hydrochloride.

From the analysis above, we would conclude that the topical anaesthetic agent that had least effect on tear quantity was $2 \%$ xylocaine hydrochloride, therefore, in this comparative analysis of the three topical anaesthetic agents, $2 \%$ xylocaine hydrochloride is recommended as topical anaesthetic agent of choice for optometric practice. For tear film stability, $0.5 \%$ tetracaine hydochloride gave a longer stability time of tear film, therefore, it is recommended as the anaesthetic of choice for patients with keratoconjunctivitis sicca because it will enhance their tear film stability. Eye care practitioners are discouraged from using proparacaine as a topical anaesthetic in patients with dry eye syndrome because this may worsen their dry eye symptoms of grittiness and burning sensation. 
Table 1: Mean tear quantity and mean tear stability of topical anaesthetic agents

\begin{tabular}{lll}
\hline Types of anaestheticagent & Mean tear Quantity $(\mathbf{m m})$ & Mean tear stability (secs) \\
\hline Control & $21.87 \pm 11.70$ & $22.63 \pm 10.92$ \\
\hline $2 \%$ xylocaine hydrochloride & $20.13 \pm 9.37$ & $21.40 \pm 7.40$ \\
$0.5 \%$ proparacaine hydrochloride & $14.07 \pm 7.61$ & $16.23 \pm 6.62$ \\
\hline $0.5 \%$ tetracaine hydrochloride & $16.10 \pm 2.85$ & $33.63 \pm 12.60$ \\
\hline
\end{tabular}

\section{REFERE N C E S}

1. Patel, S., Laidlaw, S., Mathewson, L., McCollum, L. and Nicholson, C. (1991): Iris color and the influence of local anaesthetic on precorneal tear film stability. Acta Ophthalmol. (kbh), 69:387-92.

2. Sharon, J. K. (2007): Lidocaine/tetracaine Peel. J. Anaesth. Clin. Pharamacol, 23 (3): 279-82.

3. Holly, F. J. and Lemp, M. A. (1977): Tear physiology and dry eyes. Surv. Ophthalmol, 22:69-87.

4. Prydal, J. I., Artal, P. and Woon, H. (2006): Study of human Precorneal tear film thickness structure using laser inferometry. Invest. Ophthalmol. Vis. Sci, 33(6):91-2.

5. Lemp, M. A., Holly, F. J. and Iwata, S. (1970): The precorneal tear Film. Arch Ophthalmol, 83:70-89.

6. Van Haeringen, N. J. (1981): Clinical biochemistry of Tears.

Surv. Ophthalmol; 26: 79-85.

7. Lemp, M. A. and Hamil, F. A. (1973): Factors effecting tear film break up in normal eyes. Arch. Ophthalmol, 89:103-5.

8. Lambert, D. V., Foster, C. S. and Perry, H. D. (1979): Schirmer test after topical anaesthesia and the tear meniscus height in normal eyes. Arch. Ophthalmol.97: 1082-5.

9. Merger, L. S. and Bron, A. J. (1985): A noninvasive instrument for clinical assessment of precorneal tear film stability. Curr. Eye Res, 4(1): 7-9.

10. Jordan, A. and Baum, J. (1980): Basal tear flow: Does it exist? Ophthalmol. (Rochester), 87: 920-30.

11. Cho, P. and Brown, B. (1995). The effect of benoxinate on the Tear stability of Hong Kong Chinese. Physiol. Opt, 15(4): 299-304. 